\title{
The likely fate of hybrids of Bactrocera tryoni and Bactrocera neohumeralis
}

\author{
N Pike 1,3 WYS Wang ${ }^{2}$ and A Meats ${ }^{1}$ \\ ${ }^{1}$ Fruit Fly Research Centre, School of Biological Sciences, University of Sydney, 2006, Australia; ${ }^{2}$ Cambridge Institute of Medical \\ Research, University of Cambridge, Cambridge CB2 2XY, UK
}

\begin{abstract}
Bactrocera tryoni (Froggatt) and B. neohumeralis (Hardy) (Diptera: Tephritidae) are sympatric species which hybridise readily in the laboratory yet remain distinct in the field. $B$. tryoni mates only at dusk and $B$. neohumeralis mates only during the day, but hybrids can mate at both times. We investigated the inheritance of mating time in successively backcrossed hybrid stocks to establish whether mating with either species is more likely. The progeny of all backcrosses to $B$. tryoni mated only at dusk. The majority of the progeny of the first and a minority of the progeny of the second
\end{abstract}

backcross to $B$. neohumeralis also mated at dusk, but the third successive $B$. neohumeralis backcross produced flies that mated only during the day. This trend towards dominance of the $B$. tryoni trait was also reflected in a diagnostic morphological character. We discuss the possible genetic background for these phenomena and propose that unidirectional gene flow might explain how the two species remain distinct in the face of natural hybridisation.

Heredity (2003) 90, 365-370. doi:10.1038/sj.hdy.6800253

Keywords: tephritidae; fruit fly; hybridisation; mating time; reproductive isolation

\section{Introduction}

Bactrocera tryoni and B. neohumeralis are sibling species of pestiferous fruit fly that are widespread on Australia's eastern coast. The species are sympatric, with the geographic distribution of $B$. neohumeralis being wholly contained within the greater one of B. tryoni (Birch and Vogt, 1970; Drew, 1978; Osborne et al, 1997). Biologists have paid much attention to the species because, despite their propensity to produce viable, fertile hybrids in the laboratory, they maintain their integrity in the field.

Difference in mating time has repeatedly been proposed as an explanation for the maintenance of the species' reproductive isolation (eg Wolda, 1967; Vogt, 1977; Drew, 1989). B. tryoni mates almost exclusively at dusk in dim light (Tychsen and Fletcher, 1971; Smith, 1979) and B. neohumeralis mates during the middle of the day in bright light (Gibbs, 1965; Gee, 1969). Hybrids can mate during the last part of the day as well as at dusk (Smith, 1979; Pike and Meats, 2002).

Mating activity appears to be controlled by a circadian rhythm that is synchronised to external conditions (Tychsen and Fletcher, 1971). Smith's (1979) studies of behaviour under conditions of constant lighting led him to suggest that the same rhythm or 'clock' is shared by both parent species and their hybrids. He proposed that only the 'alarm setting' may differ. Smith (1979) demonstrated that, under constant lighting conditions (and

Correspondence: N Pike, Laboratoire d'Ecologie, Ecole Normale Supérieure, 46 rue d'Ulm, Paris 75005, France.

E-mail: nathan.pike.1998@pem.cam.ac.uk.

${ }^{3}$ Current address: Laboratoire d'Ecologie, Ecole Normale Supérieure, 46 rue d'Ulm, Paris 75005, France.

Received 5 September 2002; accepted 17 January 2003 regardless of whether these were of daytime intensity or dusk intensity), the mating pattern of the $F_{1}$ and of the first $B$. tryoni backcross was indistinguishable from that of B. tryoni. Under similar conditions, he showed that the period of mating in the first $B$. neohumeralis backcross commenced much earlier but continued at a low level so that it fully overlapped those of the other groups. Pike and Meats (2002) report that, under glasshouse conditions with natural light, the mating periods of $F_{1}$ hybrids overlapped with the periods of the parent species, and that matings between hybrids and both the parent species did occur.

The morphological character by which the two species may be distinguished most reliably is the colour of the humeral calli. (The humeral calli are dorsoanterior sclerites of the thorax and appear as obvious 'shoulder pads' just posterior to the head.) B. tryoni has yellow calli, B. neohumeralis has brown calli (Perkins, 1933; May, 1963; Drew, 1989), and hybrids have calli with a central yellow patch surrounded by brown (Gibbs, 1968; Birch and Vogt, 1970; Vogt, 1977). Bateman (1958) reported that the flies with hybrid-like calli represented a small proportion of his field collections. Birch and Vogt (1970) made a detailed study of callus-colour variants and concluded that the trait was continuously variable, although vastly more common in its two extreme forms.

By studying the variation in the colour of the humeral calli, Gibbs (1968) found evidence for some hybridisation in the field. He reported that just over half of the B. tryoni females sampled from the zone of sympatry gave rise to at least some offspring with intermediate callus types and he devised an evidence-based model that allowed him to infer the male parentage of the offspring of wild female flies. He calculated that approximately $5 \%$ of 
$B$. tryoni females mated with intermediate males and that matings with $B$. neohumeralis did occur, but were exceedingly rare. Analogous results were inferred for $B$. neohumeralis females. Gibbs (1968) concluded that, whether intermediate-type flies are hybrids or otherwise, they are likely to mate with the parent species they resemble more closely. In contrast to Gibbs, Wolda (1967) opined that, based on comparisons with laboratory-bred flies, the wild-caught flies with intermediate callus types may not be $F_{1}$ hybrids but may be variants of one or the other species. He also reported on selection experiments that produced $B$. tryoni flies with calli similar to those of $B$. neohumeralis and $B$. neohumeralis flies with calli similar to those of B. tryoni. (The scant data available suggest that these flies continued to mate at their normal times.)

If significant introgression does take place, then the question arises as to how the integrity of the two species is maintained (Morrow et al's (2000) study of nuclear and mitochondrial DNA markers reports on the remarkably close genetical similarity between these two species and highlights the fact that this similarity persists despite their different behaviour and appearance). A clue is provided by the results of Smith (1979) on backcrosses between $F_{1}$ hybrids and the parent species. These show that each backcross yields a phenotype with a mating time in constant light that is similar to that of the parent species in the backcross. Thus, a backcross to $B$ neohumeralis would produce individuals that would mate at a time similar to $B$. neohumeralis and would be likely to backcross again to this species. Analogous considerations would apply if the initial backcross was to B. tryoni. However, no test on backcrosses has been done in natural (as opposed to constant) lighting conditions and the consequences for callus pattern have not been ascertained.

The role that hybrids play in genetic exchange between $B$. tryoni and B. neohumeralis is therefore potentially great, particularly if $F_{1}$ hybrids mate readily with both parent species and given that backcrossing would be virtually inevitable as hybrids would be rare compared to the numbers of flies of the parent species. By reporting on the mating behaviour and appearance of a series of backcrossed hybrid lines, we endeavour to predict the fate of genes from hybrid flies and to discuss how this may affect the integrity of the two species.

\section{Materials and methods}

\section{Laboratory conditions}

At all times, flies remained in a room with a controlled environment of $25 \pm 1{ }^{\circ} \mathrm{C}$ (mean \pm maximum fluctuation) and $65 \pm 5 \%$ relative humidity. The photophase of the $24 \mathrm{~h}$ lighting cycle had a duration of $14 \mathrm{~h}$ (which included the final hour of dusk light). The daytime light intensity was $40 \pm 5 \mu \mathrm{E} / \mathrm{m}^{2} \mathrm{~s}$ and was produced by cool white (4$100 \mathrm{~K}), 40 \mathrm{~W}$ fluorescent light tubes. The dusk light was natural and came through a double-glazed window that occupied most of the west wall. The intensity of the dusk light was about $0.3 \mu \mathrm{E} / \mathrm{m}^{2} \mathrm{~s}$ or lower. The change from daylight to dusklight was instantaneous. This quick, rather than gradual, change does not affect the flies mating behaviour (Barton Browne, 1957; Tychsen and Fletcher, 1971). Other environmental conditions and culture techniques have been described by Bateman (1967).

\section{Flies}

One $\mathrm{F}_{1}$ hybrid stock (A) was produced by crossing $B$. tryoni females with $B$. neohumeralis males, and the converse procedure produced the other stock (B). These stocks were used to find two replicate lines that were backcrossed to each parent species without further regard to sex. A concise notation that used the first letter of each species' name was used to represent the successive backcrosses conducted. The $F_{1}$ hybrids were denoted by NT, the progeny of the first backcross to $B$. neohumeralis were NNT and further backcrossings gave NNNT and NNNNT. The analogous B. tryoni backcross series was maintained only until the NTTT generation as mating preferences were constant by this stage.

\section{Mating timing tests}

Adult flies were sexed 3-5 days after emerging from their puparia. The sexes were caged separately and allowed 3 weeks to mature. Males and females were then combined in a single cage and observed every $15 \mathrm{~min}$ from 10:00 h until the fall of darkness. Mating tests involved between 350 and 500 flies and lasted up to 5 days, depending on the number of matings observed. As hybrids will mate equally often with a parent species as among themselves (Pike and Meats, 2002), it was assumed that mating among individuals of the same backcrossed line provides an accurate indication of the mating that would occur had they been exposed to flies of the parent species.

\section{Callus scoring}

Pairs of flies were permanently removed from the mating tests in $20 \mathrm{ml}$ glass phials as soon as they had coupled. The total number of mating pairs formed in each previous 15-min period was recorded from 10:00. The humeral calli were later examined and scored according to a five-class system based on the amount of yellow borne. Class 0 had between 0 and $5 \%$ yellow $(0 \%$ is all brown, typical of $B$. neohumeralis). Classes $1,2,3$ and 4 had percentages of yellow in the ranges 5-40, 40-60, 6090 and 90-100, respectively. B. tryoni has calli that are 95$100 \%$ yellow and are therefore of type 4 . The typical appearance of each of these callus classes is given in Figure 1.

\section{Analysis and transformation of data}

For Figure 3, time was standardised to make visual comparisons easier. Observations commenced at 10:00, but dusk occurred at 16:30 only for the parent species, the NT and the NNT/NTT flies. In subsequent tests, the flies' time of dusk (and therefore their artificial dawn) became progressively later as the day length increased. Unlike the flies, which were always subject to a uniform photoperiod, we humans did experience seasonal changes in day length and were consequently able to subject the flies to longer tests at certain periods of the year. These longer tests were linearly adjusted (duration compressed and counts reassigned) such that each contained just 30 observation periods between commencement and darkness. The apparent time of dusk is therefore 16:30 in all tests. Flies were all subject to an identical light-dark cycle. Standardisation served merely 


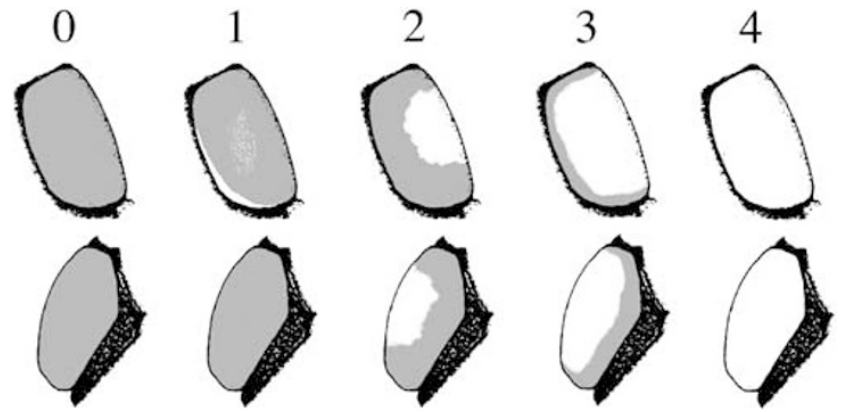

Figure 1 Numerical classification system used to score callus type. A callus typical of each class is depicted, with the top row providing a dorsal view and the bottom row providing a ventral view.

to facilitate comparison of mating distributions on a common scale (see Figure 3).

Line means and variances were calculated using untransformed data. The dominance coefficient, $K$, was calculated for the bias towards tryoni-like mating in the $\mathrm{F}_{1}(\mathrm{NT})$. The minimum number of genetic loci contributing to timing of mating was obtained with the CastleWright estimator (Lande, 1981). Epistatic effects were estimated using $\Delta$ calculated from the line means of the two parent species and their hybrid progeny (Lynch and Walsh, 1998).

A one-tailed $t$-test for samples of unequal variance was used to compare the mean mating times of $B$. tryoni and the NT flies, as they were superficially similar.

\section{Results}

\section{Mating tests}

The results for the replicate lines (A and B) were extremely similar; thus only the combined results are reported. The major trends are illustrated in Figure 1. Although only a small proportion of the $\mathrm{F}_{1}$ hybrids (NT) mated in the last hour of bright light in the day (with the majority mating at dusk), the difference between their distribution and that of $B$. tryoni was highly significant $(t=6.7778,407 \mathrm{df}, P<0.0001)$. Mating in the first $B$. neohumeralis backcross generation (NNT) started in the late afternoon and peaked at dusk. Mating in the NNNT generation commenced earlier in the day and dusk maters were in the minority. Mating in the NNNNT backcross occurred exclusively during the day.

All mating in the B. tryoni backcrosses (NTT and NTTT) occurred at dusk and was indistinguishable from the mating of B. tryoni.

\section{Quantitative analysis}

The dominance coefficient for the tryoni-like mating behaviour of the $F_{1}$ was 0.90 . The absence of any neohumeralis-like mating in the NNT indicates that more than one locus contributes to the distribution of mating time. Castle-Wright calculations indicated the involvement of a minimum of three loci. The epistatic estimator, $\Delta$, was 0.14 and not significantly different from zero $(P>0.05)$, suggesting that epistatic interactions are unlikely to play a major role. All the aforementioned calculations were repeated using time transformations to equalise the duration of the parental mating periods and
Fate of Bactrocera hybrids

$\mathrm{N}$ Pike et al

the variance of their mating distributions, and similar results were obtained.

\section{Callus scoring}

No difference in callus type was detected between the sexes or between the lines of any backcross. These groups were thus pooled for analysis and presentation. The general pattern of the results is given in Figure 2. The callus type of day-mated NNT flies (1.72 \pm 0.12 ; mean \pm SE) was significantly different from that of duskmated NNT flies $(2.31 \pm 0.07)$. Only dusk maters were found in the lines backcrossed to $B$. tryoni. The duskmated NTT flies had significantly yellower calli $(3.52 \pm 0.03)$ than those of the dusk-mated NNT. The same significant differences were found in the NNNT and the NTTT flies. Only day maters were found in the NNNNT generation and almost all of these had brown calli (callus type $0.16 \pm 0.06$ ).

\section{Discussion}

\section{The dominance of tryoni characters}

The mating of $B$. tryoni appears to be largely dominant over that of $B$. neohumeralis, but the difference between the mating of $B$. tryoni and NT demonstrates that this dominance is not complete. The exclusive dusk mating of the first and the second backcrosses to B. tryoni (NTT and NTTT) was identical to that of $B$. tryoni. Moreover, the first backcross to $B$. neohumeralis (NNT) had a distribution of mating times that was biased towards that of $B$. tryoni, although the ability of a substantial minority of the flies to mate at daytime and high light intensities is notable. It is not until the generation of the third backcross to B. neohumeralis (NNNNT) that the mating pattern approximated that typical of the parent species. These data accord with those that Smith (1979) obtained under constant lighting conditions for mating in backcross generations equivalent to our NNT and NTT.

Callus data conform to the same trend in dominance. In the NT, tryoni-like calli were the most common, and the calli found in subsequent (dusk-mating) backcrosses to $B$. tryoni were almost always of types 3 and 4 . In contrast, all five callus types were present in the first and second repeated backcrosses to B. neohumeralis, with type 3 calli being especially prevalent in the NNT. A callus distribution which was approximated to that of $B$. neohumeralis was not attained until the third backcross (NNNNT) (Figure 3).

Some correspondence between appearance and mating time is also evident in the hybrid lines that mated both during the day and at dusk (the backcrosses to $B$. neohumeralis). The callus distributions of dusk-mated flies tended to be closer to those of $B$. tryoni and tryoni backcrosses, and were significantly different from the callus distributions of day-mated flies of the same line. Day-mated hybrids tended to have callus distributions that were somewhat closer to that of $B$. neohumeralis. Flies that resembled a parent species in mating behaviour thus tended also to resemble that parent species in appearance.

The genetic background to the 'mating time' trait Our QTL analysis is useful in demonstrating the general principles that apply to the inheritance of mating time 

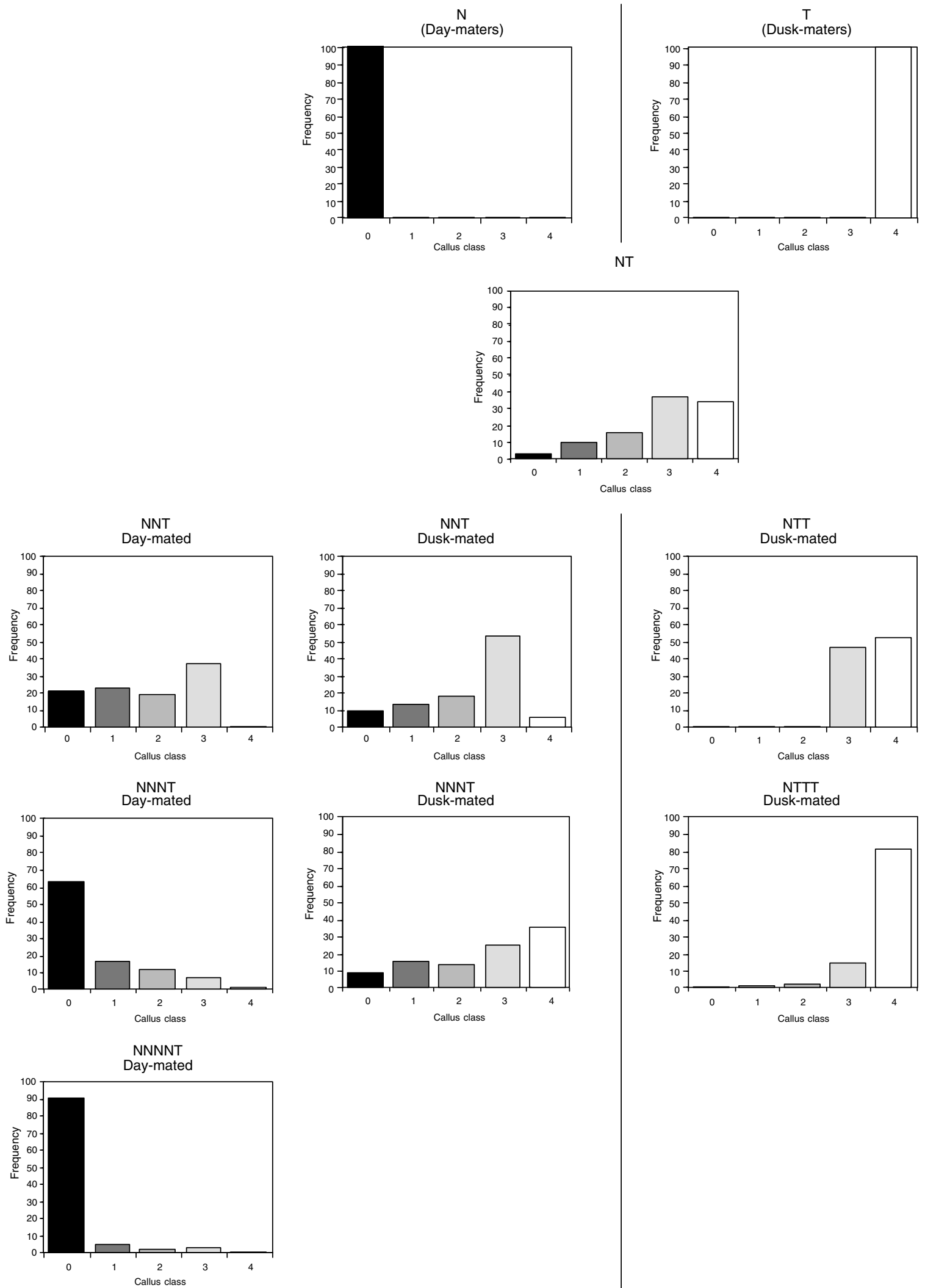

Figure 2 Frequency of callus types (described in Figure 1) in the mated progeny of successive backcrosses to B. neohumeralis and B. tryoni. The frequencies of callus types in the parent species $(\mathrm{N}, \mathrm{T})$ and $\mathrm{F}_{1}$ hybrids $(\mathrm{NT})$ are also included for reference. Day-mated NNT flies had calli that were significantly different from those of dusk-mated NNT flies. In addition, dusk-mated NTT flies had significantly yellower calli than those of the dusk-mated NNT. These significant differences occur again in the NNNT and NTTT. Further details are provided in the text. 


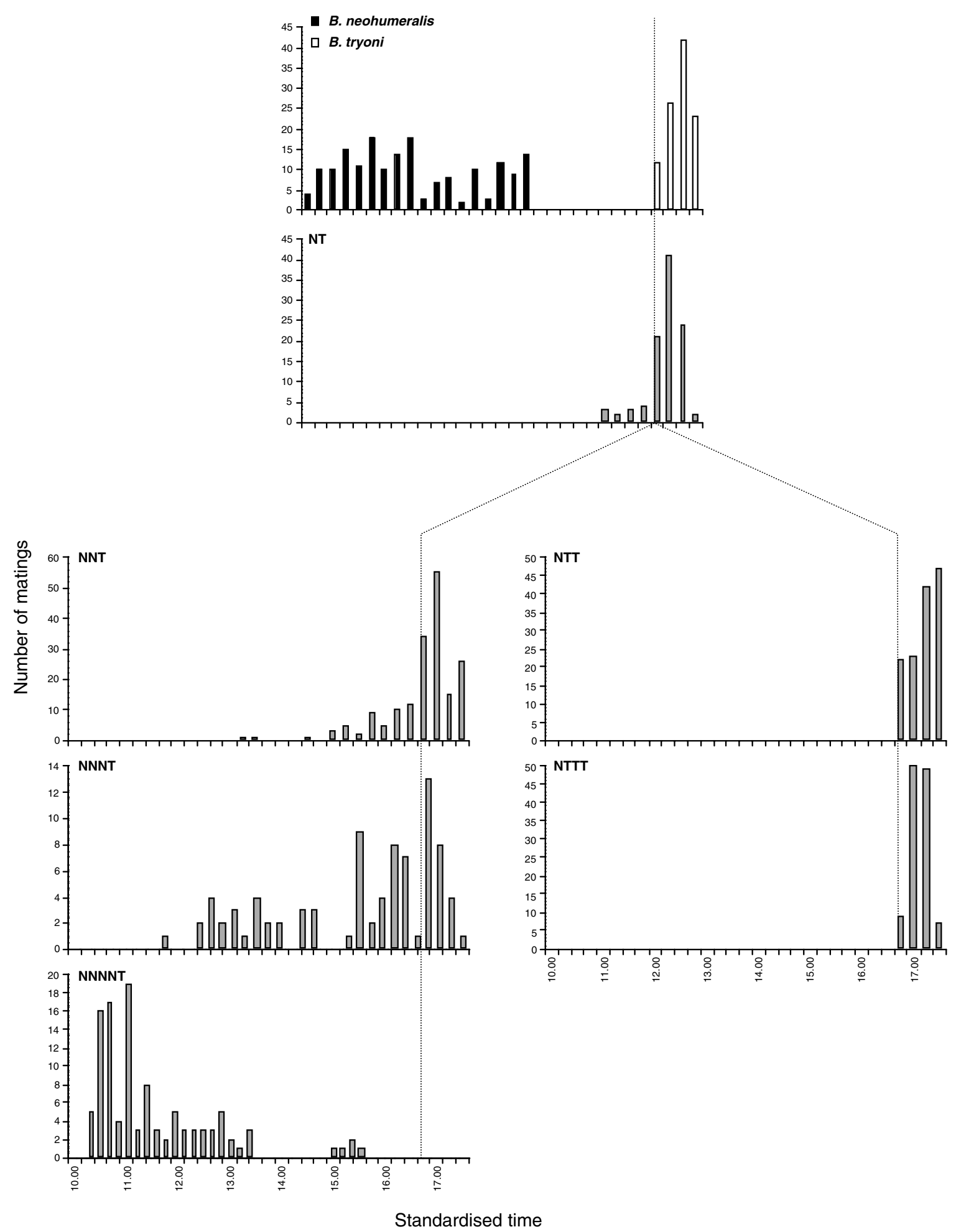

Figure 3 Distribution of mating times in B. tryoni and B. neohumeralis, $\mathrm{F}_{1}$ hybrids (NT), backcrosses to B. neohumeralis (NNT, NNNT, NNNNT) and backcrosses to B. tryoni (NTT, NTTT). Each histogram bar represents the number of mating pairs observed in a 15-min interval; the dotted line is included for reference and indicates the onset of the dusk conditions under which the final four intervals of observation were conducted.

and the estimation that, at the very least, three loci are involved in its determination is reasonable. Its results should, however, be treated with caution. Under the current experimental protocol (and, indeed, in nature), the two factors that influence the trait, time of day and light intensity, are covariant to one another. Previous experiments under conditions of constant optimal light (eg Tychsen and Fletcher, 1971; Smith, 1979) provide methods by which the effects of these factors might be separated and have shown that the period acceptable for 
mating among $B$. tryoni can be just as long as that commonly seen in $B$. neohumeralis (ie $>7 \mathrm{~h}$ ).

Although we use a lighting regime that is very similar to the natural one, another (necessary) aspect of our protocol means that our mating distributions are probably only good approximations of those that occur in the field. Depriving the sexes of access to one another must increase their eagerness to mate, and the mating peaks that occur just subsequent to the commencement of tests containing day-mating flies are evidence of this. Despite the fact that our testing procedure is not ideal for plotting precise natural mating distributions, it does inadvertently support the theory for preservation of specific integrity outlined below (unidirectional gene flow). As the eager virgins are first subject to a period of at least $6.5 \mathrm{~h}$ of daytime and its associated high light intensity, that majority of flies of the early B. neohumeralis backcrosses which abstains from mating until dusk is doubly confirmed in its preference for dusk mating.

\section{The integrity of the species}

Lewontin and Birch (1966) conjectured that introgression from $B$. neohumeralis could be responsible for providing the genetic variability that permitted B. tryoni to expand its geographic range. In addition, they speculated that naturally occurring intermediate character states are indeed attributable to interspecific genetic exchange, but are not necessarily the direct result of an interspecific mating.

The backcrosses' tendency to retain B. tryoni-like mating patterns is likely to have profound consequences for the maintenance of specific integrity in the two species. Although it appears that $F_{1}$ hybrids can mate with either parent species under some circumstances (Pike and Meats, 2002), this may not necessarily result in balanced bidirectional introgression - even if the frequencies of the parent species were equal. If $F_{1}$ hybrids do occur naturally and do mate with one or both parent species in the field, our current results suggest that these hybrids, as well as flies from any backcross to $B$. neohumeralis, will mate predominantly with $B$. tryoni because there is a bias towards dusk mating in the hybrids and the progeny of such backcrosses. The current study emphasises the extent to which various backcrosses prefer to mate at dusk. If we consider this natural disposition in conjunction with the observations that only $B$. tryoni has pooled courtship in the form of leks of pheromone-releasing males and only B. tryoni mates intensively at dusk, (Tyschen, 1977), we may predict that most hybrids as well as their descendants are destined to mate with B. tryoni. This largely unidirectional flow of neohumeralis genes into the tryoni pool is likely to be a pivotal factor in allowing the continuing integrity of the two species. As this flow is from the species that occurs exclusively in sympatric populations (B. neohumeralis) to the species that also occurs in large allopatric populations (B. tryoni), even weak selection against neohumeralis genes in tryoni populations could well suffice to maintain B. tryoni's integrity. If the direction of gene flow were to occur predominantly in the opposite direction, considerably stronger selection would no doubt be required to maintain B. neohumeralis's integrity.

\section{References}

Barton Browne L (1957). An investigation of the low frequency of mating of the Queensland fruit fly Strumeta tryoni (Frogg.). Aust J Zool 5: 159-163.

Bateman MA (1958). Ecological Adaptations in Geographic Races of the Queensland Fruit Fly Dacus (Strumeta) tryoni Frogg. PhD, University of Sydney.

Bateman MA 1967). Adaptations to temperature in geographic races of the Queensland fruit fly, Dacus (Strumeta) tryoni. Aust J Zool 15: 1141-1161.

Birch LC, Vogt WG (1970). Plasticity of taxonomic characters of the Queensland fruit flies Dacus tryoni and Dacus neohumeralis (Tephritidae). Evolution 24: 320-343.

Drew RAI (1978). Section I. Taxonomy. In: Drew RAI, Hooper GHS, Bateman MA (eds). Economic Fruit Flies of the South Pacific Region, Queensland Department of Primary Industries: Brisbane., pp 1-94.

Drew RAI (1989). The tropical fruit flies (Diptera: Tephritidae; Dacinae) of the Australasian and Oceanian regions. Memoirs Queensl Museum 26: 1-521.

Gee JH (1969). Effect of daily synchronisation of sexual activity and mating success in laboratory populations of two species of Dacus (Diptera: Tephritidae). Aust J Zool 17: 619-624.

Gibbs GW (1965). Comparative ecology and sexual isolation of two species of Dacus. PhD, University of Sydney.

Gibbs GW (1968). The frequency of interbreeding between two sibling species of Dacus (Diptera) in wild populations. Evolution 22: 667-683.

Lande R (1981). The minimum number of genes contributing to quantitative variation between and within populations. Genetics 99: 541-553.

Lewontin RC, Birch LC (1966). Hybridization as a source of variation for adaptation to new environments. Evolution 20: 315-336.

Lynch M, Walsh B (1998). Genetics and Analysis of Quantitative Traits. Sinauer: Sunderland, MA.

May AWS (1963). An investigation of fruit flies (Trypetidae: Diptera) in Queensland. Queensland J Agric Sci 20: 1-82.

Morrow J, Scott L, Congdon B, Yeates D, Frommer M, Sved J (2000). Close genetic similarity between two sympatric species of tephritid fruit fly reproductively isolated by mating time. Evolution 54: 899-910.

Osborne R, Meats A, Frommer M, Sved JA, Drew RAI, Robson MK (1997). Australian distribution of 17 species of fruit flies (Diptera: Tephritidae) caught in cue lure traps in February 1994. Aust J Entomol 36: 45-50.

Perkins FA (1933). New Australian Trypetidae with notes on previously described species. Proc $R$ Soc Queensl 45: 41-44.

Pike N, Meats A (2002). Potential for mating between Bactrocera tryoni (Froggatt) and B. neohumeralis (Hardy) (Diptera: Tephritidae). Aust J Entomol 41: 70-74.

Smith PH (1979). Genetic manipulation of the circadian clock's timing of sexual behaviour in the Queensland fruit flies, Dacus tryoni and Dacus neohumeralis. Physiol Entomol 4: 71-78.

Tychsen PH (1977). Mating behaviour of the Queensland fruit fly, Dacus tryoni (Diptera: Tephritidae), in field cages. J Aust Entomol Soc 16: 459-465.

Tychsen PH, Fletcher BS (1971). Studies on the rhythm of mating in the Queensland fruit fly, Dacus tryoni. J Insect Physiol 17: 2139-2156.

Vogt WG (1977). A re-evaluation of introgression between Dacus tryoni and Dacus neohumeralis (Diptera: Tephritidae). Aust J Zool 25: 59-69.

Wolda H (1967). Reproductive isolation between two closely related species of the Queensland fruit fly, Dacus tryoni (Frogg.) and D. neohumeralis Hardy (Diptera: Tephritidae). II. Genetic variation in humeral callus pattern in each species as compared with laboratory-bred hybrids. Aust J Zool 15: 515539. 